\title{
Author Correction: Weighing Scale-Based Pulse Transit Time is a Superior Marker of Blood Pressure than Conventional Pulse Arrival Time
}

\author{
Stephanie L.-O. Martin ${ }^{1}$, Andrew M. Carek ${ }^{2}$, Chang-Sei Kim ${ }^{1}$, Hazar Ashouri², Omer T. Inan ${ }^{2}$, \\ Jin-Oh Hahn ${ }^{1} \&$ Ramakrishna Mukkamala ${ }^{3}$ \\ Correction to: Scientific Reports https://doi.org/10.1038/srep39273, published online 15 December 2016
}

This Article contains errors.

Figure 6 is incorrect due to an error when calculating linear regression, where $\mathrm{x}$ was inadvertently set as the dependent variable and $\mathrm{y}$ as the independent variable.

This error does not cause a change to the main conclusion of this Article, but does result in some changes to the detailed interpretation of the results.

The correct Figure 6 and its accompanying legend appear below as Figure 1.

As a result, in the Abstract,

"Scale PTT tracked the diastolic BP changes well, with correlation coefficient of $-0.80 \pm 0.02$ (mean \pm SE) and root-mean-squared-error of $7.6 \pm 0.5 \mathrm{mmHg}$ after a best-case calibration. Conventional PAT was significantly inferior in tracking these changes, with correlation coefficient of $-0.60 \pm 0.04$ and root-mean-squared-error of $14.6 \pm 1.5 \mathrm{mmHg}(\mathrm{p}<0.05)$. Scale PTT also tracked the systolic BP changes better than conventional PAT but not to an acceptable level."

should read:

"Scale PTT tracked the diastolic and systolic BP changes well, with correlation coefficients of $-0.80 \pm 0.02$ (mean \pm SE) and $-0.80 \pm 0.04$ and root-mean-squared-errors of $5.7 \pm 0.4$ and $8.5 \pm 0.7 \mathrm{mmHg}$ after best-case calibrations. Conventional PAT was significantly inferior in tracking these changes, with corresponding correlation coefficients of $-0.60 \pm 0.04$ and $-0.66 \pm 0.03$ and root-mean-squared-errors of $7.1 \pm 0.6$ and $10.1 \pm 0.7 \mathrm{mmHg}$ $(\mathrm{p}<0.05$ or 0.10$)$."

In the Results section,

"Scale PTT yielded a good diastolic BP RMSE $(7.6 \pm 0.5 \mathrm{mmHg})$ that was $48 \%$ lower than that of conventional PAT. Scale PTT also provided a systolic BP RMSE that was $36 \%$ lower than that of conventional PAT. However, despite also being best-case, the systolic BP RMSE of scale PTT was still not good (11.8 $\pm 1.6 \mathrm{mmHg})$."

${ }^{1}$ Department of Mechanical Engineering, University of Maryland, College Park, MD, USA. ${ }^{2}$ School of Electrical and Computer Engineering, Georgia Institute of Technology, Atlanta, GA, USA. ${ }^{3}$ Department of Electrical and Computer Engineering, Michigan State University, East Lansing, MI, USA. Stephanie L.-O. Martin, Andrew M. Carek and ChangSei Kim contributed equally. Correspondence and requests for materials should be addressed to O.T.I. (email: omer. inan@ece.gatech.edu) or J.-O.H. (email: jhahn12@umd.edu) or R.M. (email: rama@egr.msu.edu) 

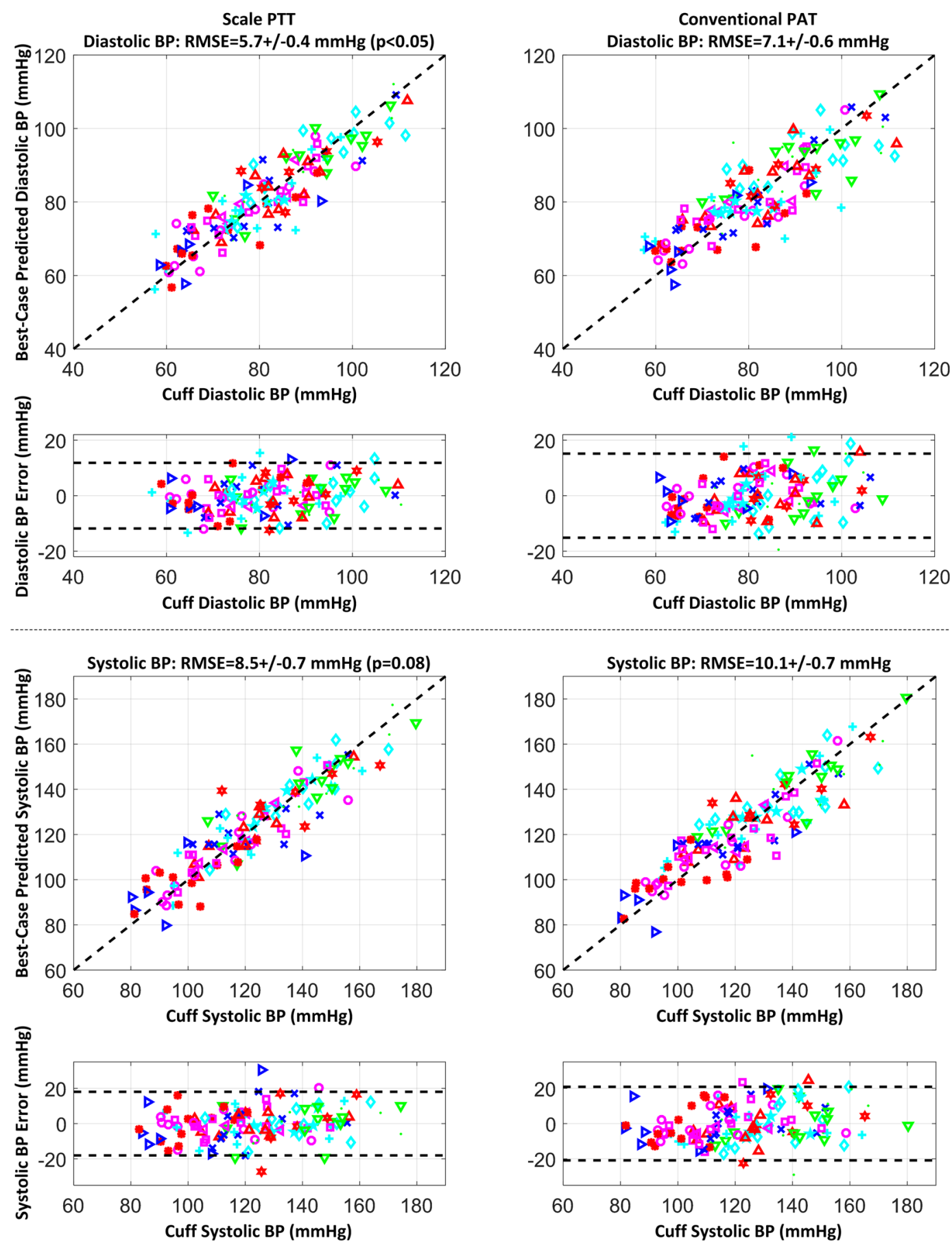

Figure 1. Correlation plots of predicted BP via scale PTT and via conventional PAT after best-case calibration for each time delay versus cuff BP and Bland-Altman plots of the errors between the predicted and measured $\mathrm{BP}$ versus cuff $\mathrm{BP}$ pooled over all the subjects, along with the group average RMSEs. The different symbols correspond to each of the subjects. Scale PTT yielded lower diastolic and systolic BP RMSEs than conventional PAT..

should read:

"Scale PTT yielded a good diastolic BP RMSE $(5.7 \pm 0.4 \mathrm{mmHg})$ that was $20 \%$ lower than that of conventional PAT. Scale PTT also provided a good systolic BP RMSE $(8.5 \pm 0.7 \mathrm{mmHg})$ that was $15 \%$ lower than that of conventional PAT."

In the Discussion section, 
"Scale PTT, which is extracted at the diastolic level of the waveforms (see Fig. 2A), tracked the diastolic BP changes fairly well, with a correlation coefficient of $-0.80 \pm 0.02$ (see Figs 4 and 5) and a best-case RMSE of $7.6 \pm 0.5 \mathrm{mmHg}$ after calibration with the reference cuff BP (see Fig. 6). The corresponding quantitative metrics offered by conventional PAT were $-0.60 \pm 0.04$ and $14.6 \pm 1.5 \mathrm{mmHg}$ (see Figs $4,5,6$ ). The elimination of PEP and perhaps the mitigation of the impact of smooth muscle contraction in scale PTT led to these 30-50\% improvements (see Fig. 3). Scale PTT also afforded superior tracking of the systolic BP changes compared to conventional PAT (see Figs 4 and 6). However, even with the best possible calibration, scale PTT could only yield a systolic BP RMSE of $11.8 \pm 1.6 \mathrm{mmHg}$. In sum, scale PTT provided good tracking of diastolic BP changes, whereas conventional PAT did not track the changes in either diastolic or systolic BP with a level of accuracy that is close to acceptable."

should read:

"Scale PTT, which is extracted at the diastolic level of the waveforms (see Fig. 2A), tracked the diastolic BP changes well, with a correlation coefficient of $-0.80 \pm 0.02$ (see Figs 4 and 5) and a best-case RMSE of $5.7 \pm 0.4 \mathrm{mmHg}$ after calibration with the reference cuff BP (see Fig. 6). The corresponding quantitative metrics offered by conventional PAT were $-0.60 \pm 0.04$ and $7.1 \pm 0.6 \mathrm{mmHg}$ (see Figs $4,5,6$ ). The elimination of PEP and perhaps the mitigation of the impact of smooth muscle contraction in scale PTT led to these $20-33 \%$ improvements (see Fig. 3). Because the systolic BP changes paralleled the diastolic BP changes in this study (see Fig. 3), scale PTT was also able to track the systolic BP changes fairly well and better than PAT by 15-20\% (see Figs 4 and 6). The diastolic and systolic BP RMSE reduction of $1.5 \mathrm{mmHg}$ afforded by scale PTT may appear modest. However, based on the definition of the correlation coefficient and the fact that the bias error is near zero for best-case calibration, the RMSE here scales with the magnitude of the BP change (i.e., RMSE $=1-r 2 \sigma B P$, where $r$ is the correlation coefficient and $\sigma B P$ is the standard deviation of $\mathrm{BP}$ ). In this study, the standard deviation of BP due to the interventions was not large (e.g., about $10 \mathrm{mmHg}$ for diastolic BP, as shown in Fig. 3). Hence, the RMSE reduction afforded by scale PTT may become substantial with a more significant BP range. In sum, scale PTT tracked the BP changes with a level of accuracy that was good overall and better than conventional PAT.'

(i) Open Access This article is licensed under a Creative Commons Attribution 4.0 International License, which permits use, sharing, adaptation, distribution and reproduction in any medium or format, as long as you give appropriate credit to the original author(s) and the source, provide a link to the Creative Commons license, and indicate if changes were made. The images or other third party material in this article are included in the article's Creative Commons license, unless indicated otherwise in a credit line to the material. If material is not included in the article's Creative Commons license and your intended use is not permitted by statutory regulation or exceeds the permitted use, you will need to obtain permission directly from the copyright holder. To view a copy of this license, visit http://creativecommons.org/licenses/by/4.0/.

(c) The Author(s) 2018 